\title{
sciendo
}

Current Issues in Pharmacy and Medical Sciences

Formerly ANNALES UNIVERSITATIS MARIAE CURIE-SKLODOWSKA, SECTIO DDD, PHARMACIA

journal homepage: http://www.curipms.umlub.pl/

\section{Histopathological comparison of the salivary glands' acini and striated ducts after experimental prolonged daily administration of oral ubiquinone doses in rats}

\author{
Ali Ghanim Abdullah ${ }^{1}$, Ban Ismael Sedeeq², Marwan Saad Azzubaidi ${ }^{3 *}$ (e) \\ ${ }^{1}$ Department of Oral and Maxillofacial Surgery, College of Dentistry, Tikrit University, Iraq \\ ${ }^{2}$ Department of Basic Sciences, College of Dentistry, Tikrit University, Iraq \\ ${ }^{3}$ Pharmacology Unit, Faculty of Medicine, Universiti Sultan Zainal Abidin, Kuala Terengganu, Malaysia
}

\section{ARTICLE INFO \\ Received 31 July 2021 \\ Accepted 30 October 2021}

\section{Keywords:}

ubiquinone,

salivary gland,

acini,

striated ducts,

diameter.

\begin{abstract}
Also called coenzyme Q10 (CoQ10), Ubiquinone is a vitamin-like endogenously produced factor essential for Adenosine triphosphate (ATP) mitochondrial production. Several research studies have reported that the exogenous supplementation of CoQ10 can lead to excessive salivation, especially in patients complaining of dry mouth. The objective of this study was to investigate the effect of long-term daily use of CoQ10 on the salivary glands in experimental animals by comparing the diameters of the glandular acini and striated ducts of a CoQ10-treated group and a control group. Twenty-five white albino rats were randomly divided into two groups; the control group consisted of 10 rats, while the CoQ10-treated group comprised 15 rats. The latter received daily oral treatment of $300 \mathrm{mg} / \mathrm{kg}$ CoQ10 for six weeks. Samples of the parotid, submandibular and sublingual glands were then dissected and examined histologically for comparative measurement of the diameters of the glands' acini and striated ducts. The CoQ10 treated group had mean diameters of the serous acini for the parotid $(79.8 \pm 11.2 \mu \mathrm{m})$ and submandibular $(81.07 \pm 13.5 \mu \mathrm{m})$ glands that were significantly higher $(\mathrm{P}<0.05)$ than their diameters in the control group $(67.5 \pm 8.4 \mu \mathrm{m}$ and $73.3 \pm 13.8 \mu \mathrm{m})$, respectively. However, the difference was not statistically significant when comparing the diameters of striated ducts of the CoQ10-treated group and the control group. Continuous and prolonged exposure to exogenous ubiquinone may cause hypertrophic dilation of the acini within the salivary glands, namely the parotid and submandibular glands, which might be the underlying mechanism for excessive salivation. This can be considered a reversible adaptive response.
\end{abstract}

\section{INTRODUCTION}

Co-enzyme (CoQ10), also known as 'ubiquinone' (the oxidized form of CoQ10), is a lipid-soluble vitamin-like benzoquinone derivative. Being structurally similar to vitamin $\mathrm{K}$, ubiquinone is found in the inner mitochondrial membrane of most eukaryotic cells. Besides its free radical scavenging activity during oxidative phosphorylation, its function is to act as an electron acceptor and donor in the electron transport chain involved in ATP production [1].

CoQ10 is present in all organs in variable amounts, with its highest concentrations found in tissues with high energy requirements or high metabolic activity, such as the heart, kidneys, liver and muscles. CoQ10 is primarily found as ubiquinol in these tissues, and is mostly concentrated

\footnotetext{
* Corresponding author

e-mail:mazzubaidi@gmail.com
}

in the mitochondria, indicating its importance in mitochondrial function [2]. Since ubiquinone is synthesized in all organs, the body is not normally dependent on exogenous supplies of CoQ10 unless in cases of malnutrition. Although exogenous CoQ10 is well-tolerated by the human body, a variety of adverse reactions have been reported, but these were described as being infrequent and generally mild, such as decreased appetite, nausea, vomiting, diarrhea, dyspepsia and dizziness [3]. More recent animal-based research studies are indicating the possible incidence of local toxic effects resulting from the prolonged administration of ubiquinone, especially to the kidney and (less severely) the liver [4,5]. Human trials using ubiquinone have reported contrasting results by researchers using almost the same research protocol to test the effect of a specific dose of CoQ10 on salivary glands secretions. 
On the one hand, Koufuchi and his co-workers have asserted that daily oral treatment of $100 \mathrm{mg}$ CoQ10 to patients who suffered from dry mouth resulted in a significant improvement in the activity of salivary secretions [6]. On the other hand, Sekine et al. [7] demonstrated in their research study a significant correlation between plasma levels of CoQ10 and the amount of saliva secreted by the parotid glands after giving $200 \mathrm{mg}$ of ubiquinone daily to healthy volunteers. Contrastingly, Dawood and her colleagues found no histological changes on the buccal salivary glands of mice after 2 weeks of daily $200 \mathrm{mg} / \mathrm{kg}$ oral doses of ubiquinone [8].

\section{AIM}

This study aimed to make a quantitative histological assessment of the major salivary glands (parotid, submandibular and sublingual) in rats after a six-week treatment with a daily oral dose of $300 \mathrm{mg} / \mathrm{kg} \mathrm{CoQ} 10$. The parameter considered for quantification was the diameter of the three salivary glands acini and striated ducts.

\section{MATERIALS AND METHODS}

\section{Rats}

This study consisted of the use of 25 female Wistar rats whose weight ranged from 220-270 g. All animals were housed in metal cages in the animal care facility at the College of Veterinary Medicine in Tikrit University and kept at room temperature, with 12 hours daily exposure to light. Rats had free access to dietary food pellets and water ad libitum. The experiment was performed according to the Guidelines for the care and use of laboratory animals and the European Council Directive on 24 November 1986 for care and use of Laboratory animals (86/609/EEC). Furthermore, the local ethics committee's approval of the study protocol was obtained under the reference number (202098-0809). After an acclimatization period of 1-week, the rats were randomly split into two groups, the control group $(\mathrm{n}=10)$ and the CoQ10-treated group $(\mathrm{n}=15)$.

In addition to receiving their standard food pellets, rats in the CoQ10-treated group were orally treated with
$300 \mathrm{mg} / \mathrm{kg} /$ day of CoQ10 by oral gavage for six weeks. By the end of the course of treatment, the rats were euthanized under general anesthesia. Samples of the three salivary glands (parotid, submandibular and sublingual) were then excised to be fixed in 10\% neutral-buffered formaldehyde solution for 48 hours before processing and sectioning.

\section{Samples processing}

With graded alcohol concentrations, processing of samples was done till embedding in paraffin wax blocks. Herein, 5- $\mu \mathrm{m}$ sections were prepared under the microtome to be stained later with hematoxylin \& eosin (H\&E) as described previously by Abdullah and co-authors [4]. An Olympus light microscope was utilized to observe and quantify the histological parameters.

\section{Measuring technique}

Ten microscopic fields were considered in each slide of the control and the treated groups to identify the mean value of the diameter of salivary acini and the striated ducts as histological parameters [9]. Further analysis of the significant differences in the diameters of the control and the CoQ10-treated groups was done using student's t-test.

\section{RESULTS}

\section{Parotid glands}

Histological samples of the control group revealed the typical structure of the salivary gland consisting of serous acini, striated ducts, and interlobular ducts as shown in Figure 1A. The mean diameter of the serous acini was $67.5 \pm 8.4 \mu \mathrm{m}$ as seen in Table 1 , while the mean diameter of striated ducts was $79.9 \pm 15.2 \mu \mathrm{m}$ as explained in Table 1 .

Although sections of the parotid gland from the CoQ10treated group also showed the standard structure of serous acini, striated ducts and interlobular ducts as demonstrated in Figure 1B, the mean diameter of serous acini was significantly greater $(79.8 \pm 11.2 \mu \mathrm{m})$ than that of the control group $(\mathrm{p}<0.05)$, as shown in Table 1 . Nevertheless, the diameter of striated ducts displayed an insignificant rise which was $85.5 \pm 11.07 \mu \mathrm{m}$, as shown in Table 1 .
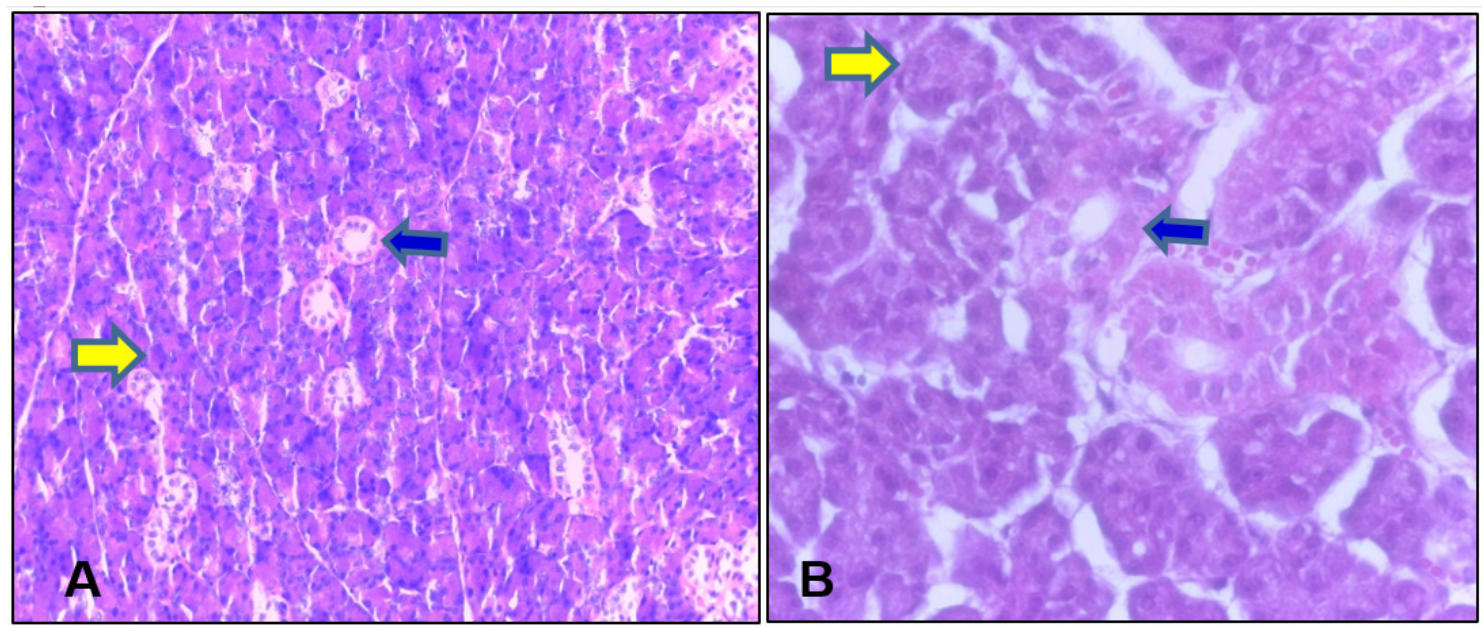

Figure 1. Photomicrographs of the parotid gland of the A) the control group at 200× magnification power, B) CoQ10-treated group at $500 \times$ magnification power. The yellow arrows are pointing at serous acini, while the blue arrows are pointing at the striated duct 


\section{Submandibular gland}

The control group sections exhibited normal morphology of serous acini, striated ducts, and interlobular ducts as seen in Figure 2A. The mean diameter of the acini was $73.3 \pm$ $13.8 \mu \mathrm{m}$ as shown in Table 1, whereas the mean diameter of striated ducts for the control group was $99.4 \pm 10.7 \mu \mathrm{m}$ as demonstrated in Table 1.

The acini of the submandibular gland of the CoQ10treated group showed an average diameter of $81.07 \pm 13.5 \mu \mathrm{m}$ that was significantly greater than that of the control group as revealed in Table 1. However, the mean diameter of the striated ducts $(97.6 \pm 12.3 \mu \mathrm{m})$ exhibited an insignificant difference, though higher than that of the control group, as demonstrated in Figure 2B and Table 1.

Table 1. Comparison of the mean \pm SD diameter of the acini and striated ducts for the control and CoQ10 treated groups for the 3 studied salivary glands

\begin{tabular}{|c|c|c|c|c|}
\hline \multirow{2}{*}{ GLAND } & \multicolumn{2}{|c|}{$\begin{array}{c}\text { Acini diameter } \\
(\mu \mathrm{m})\end{array}$} & $\begin{array}{c}\text { Striated ducts diameter } \\
(\mu \mathrm{m})\end{array}$ \\
\cline { 2 - 5 } & Control & CoQ10 & Control & CoQ10 \\
\hline Parotid & $67.5 \pm 8.4$ & $* 79.8 \pm 11.2$ & $79.9 \pm 15.2$ & $85.5 \pm 11.07$ \\
\hline Submandibular & $73.3 \pm 13.8$ & $* 81.07 \pm 13.5$ & $99.4 \pm 10.7$ & $97.6 \pm 12.3$ \\
\hline Sublingual & $118.6 \pm 26.1$ & $111.6 \pm 20$ & $124.3 \pm 12.3$ & $128.4 \pm 20$ \\
\hline
\end{tabular}

\section{Sublingual gland}

Sections of the sublingual gland of the control group, depicted in Figure 3A, revealed the normal appearance of mixed mucous acini (mean diameter $=118.6 \pm 26.1 \mu \mathrm{m})$, serous demilunes, and striated ducts (mean diameter $=124.3 \pm$ $12.3 \mu \mathrm{m}$ ) as seen in Table 1. As for the CoQ10 treated group, the histological sections portrayed normal mucous acini and striated ducts as demonstrated in Figure 3B. The diameter of the acini in the CoQ10-treated group $(111.6 \pm 20 \mu \mathrm{m})$ was insignificantly different from that of the control group, as described in Table 1. Similarly, the mean diameter of striated ducts $(128.4 \pm 20 \mu \mathrm{m})$, although higher than that of the control group, this difference was statistically insignificant, as shown in Table 1. These results reflect the favorable safety profile of the long-term use of ubiquinone daily doses on the sublingual gland structure and function.

\section{DISCUSSION}

In the current study, treating the experimental animals with $300 \mathrm{mg} / \mathrm{kg}$ /day ubiquinone orally for six weeks is considered a relatively high daily dose for a long-term duration. The purpose of using this high dose for such a prolonged duration was to elucidate the potentially toxic effects ubiquinone might produce on the salivary glands in healthy

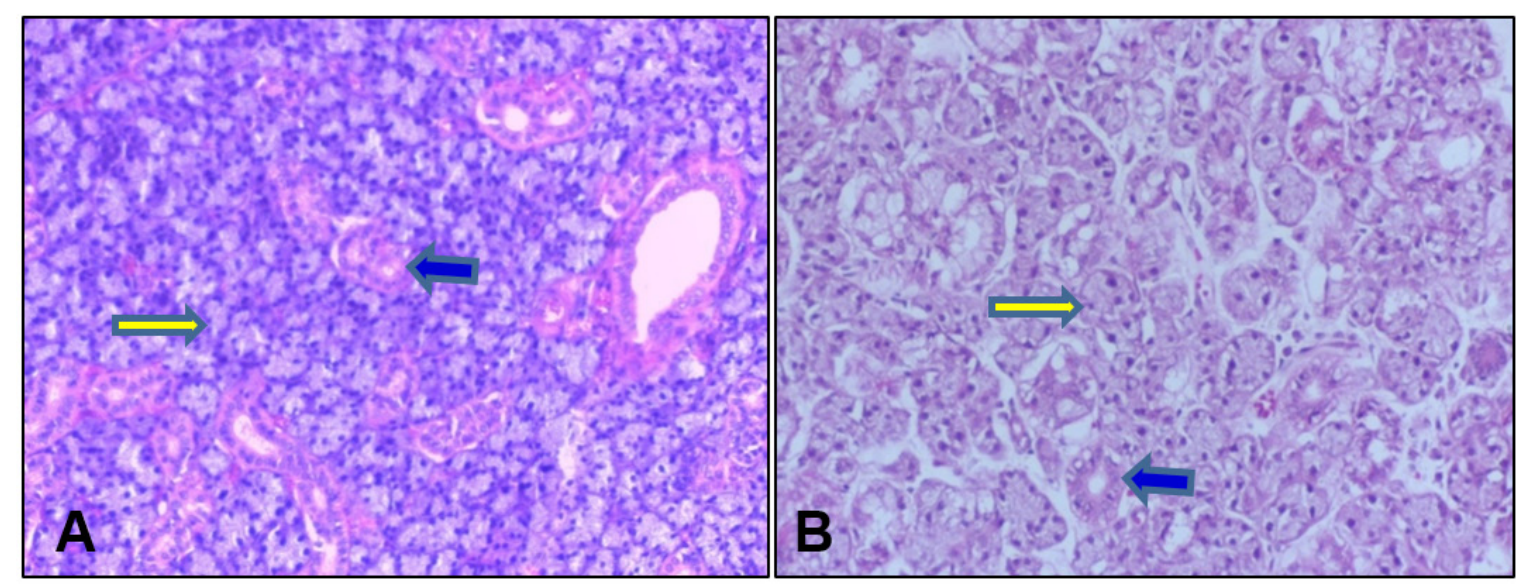

Figure 2. Photomicrographs of the submandibular glands of the A) the control group, B) CoQ10-treated group, at 200× magnification power. The yellow arrows are pointing at the acini, while the blue arrows are pointing at the striated duct

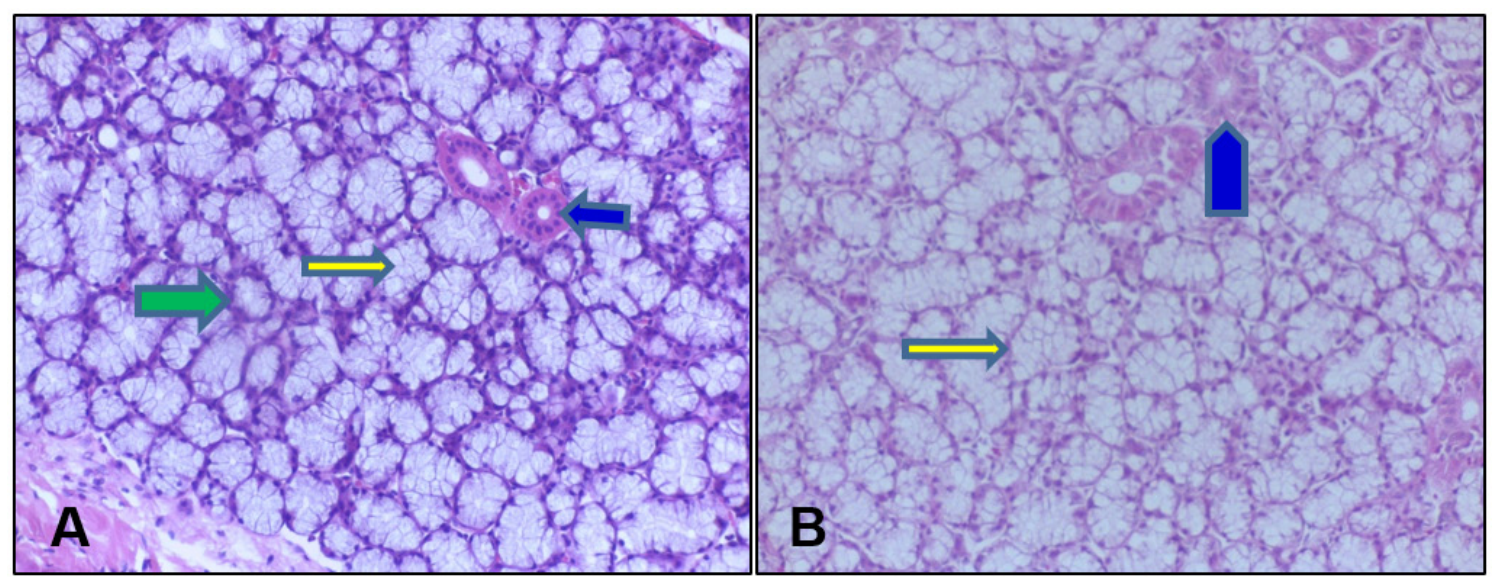

Figure 3. Photomicrographs of the sublingual glands of the A) the control group, B) CoQ10-treated group, at 200× magnification power. The yellow arrows point at the acini. The green arrow points towards the serous demilunes, while the blue arrows point at the striated duct 
experimental animals. The reason for choosing the testing dose of $300 \mathrm{mg} / \mathrm{kg} /$ day was to avoid adverse effect on liver enzymes that was reported to happen at $600 \mathrm{mg} / \mathrm{kg} / \mathrm{day}$. At such a level, female rats were reported to suffer an increase in liver enzymes with gross focal liver changes. Histopathological evaluation of the hepatic areas with focal changes revealed hepatocellular necrosis [10]. Therefore, the daily oral dose of $300 \mathrm{mg} / \mathrm{kg} \mathrm{CoQ} 10$ was selected. Histological samples of salivary glands were sectioned from rats' parotid, submandibular, and sublingual glands from both control and treated groups.

The three major salivary glands did not display profound regressive histological changes such as atrophy or degeneration of the parenchyma. This result came in agreement with outcomes of studies conducted by Deshmukh et al. [10] and another experiment undertaken in 2011 by Kitano et al. [11], except for the finding of a significant increase in diameter (hypertrophy) of serous acini in both parotid and submandibular glands in the current study. This increase in the size of acini (hypertrophy) could be attributed to several causes, such as repeated feeding with certain dietary supplements like grape skin extract or tannic acid [12], where glandular hypertrophy is considered a reversible adaptive response. Hyperlipidemia is a possible consequence of prolonged use of ubiquinone. This result was reported by Honda and coworkers [13], who documented elevated blood phosholipids levels after 13 weeks of administration of ubiquinone. Therefore, this status of ubiquinone-induced hyperlipidemia could be a contributing factor that may trigger the hypertrophy of the parotid gland's serous acini, as stated in the experiment conducted in 2011 by Daskala et al. [14]. Yet, in the present study, no signs of inflammation or fibrosis in experimental group were observed.

Other causes of hypertrophy or increase in diameter of the serous acini of the salivary glands could be the development of edema and fluid retention in the stroma and parenchyma of the gland - as revealed in a study carried out by Veiga and co-researchers. They reported an increase in the acini sizes due to ingestion of a toxic substance [15]. Therefore, concerns are raised that more extended periods of continuous exposure to high doses of ubiquinone may result in an adaptive malfunction of the salivary glands that warrants precautions. For this reason, the changes in the diameters of salivary glandular serous acini can be considered a sensitive early indicator of salivary gland functional adaptation to an external effector element, including malnutrition or specific disease conditions such as Chagas disease $[16,17]$.

A recent study reported the mean values of $36.2 \pm 2$ for the outer diameter of the submandibular glands acini of white rats [18], while in our study the mean control values were higher. The reason for this difference in the two studies could be due to using different rat species, different rat gender, as well as differences in animal weight [19].

\section{CONCLUSION}

Long-standing ingestion of ubiquinone had led the parotid and submandibular salivary glands to undergo a significant dilation resulting from hypertrophic changes in their acini ( $p<0.05$ ), but not in the striated ducts. This cellular hypertrophy of the salivary glands' acini might explain the mechanism underlying the excessive salivation noticed in human trials treated with CoQ10. Although CoQ10 is among the most popular dietary and nutritional supplements available in the market, it should be considered that CoQ10 is not yet approved by the American Food and Drug Administration as a treatment for any medical condition. Instead, it is presented as a nutritional supplement, and its manufacturing is not regulated the same way other drugs are [20].

\section{ACKNOWLEDGEMENT}

The authors are hereby expressing their appreciation to the staff of the animal house in the college of veterinary medicine in Tikrit University for sharing their experiences and efforts in handling animals during the experiment period.

\section{CONFLICT OF INTEREST}

None declared.

\section{ORCID iDs}

Marwan Saad Azzubaidi

(D)https://orcid.org/0000-0002-9118-5930

\section{REFERNCES}

1. Ferrante RJ, Andreassen OA, Dedeoglu A, Ferrante KL, Jenkins BG, Hersch SM, et al. Therapeutic effects of coenzyme Q10 and remacemide in transgenic mouse models of Huntington's disease. $J$ Neurosci. 2002;22(5):1592-9.

2. Bhagavan HN, Chopra RK. Coenzyme Q10: absorption, tissue uptake, metabolism and pharmacokinetics. Free Radic Res. 2006; 40(5):445-53.

3. Langsjoen PH, Langsjoen AM. Supplemental ubiquinol in patients with advanced congestive heart failure. Biofactors. 2008; 32(1-4):119-28.

4. Abdullah AG, Sedeeq BI, Azzubaidi MS. Histopathological nephrotoxic features of high oral doses of ubiquinone in rats. Curr Issues Pharm Med Sci. 2021;34(2):101-4.

5. Abdullah AG, Sedeeq BI, Azzubaidi MS. Histopathological changes in liver tissue after repeated administrations of an intermediate dose of co-enzyme Q10 to Wistar rats. Research J Pharm Tech. 2021;14(8):2025-8.

6. Koufuchi R, Atsuko I, Rie TYT, Kazumune A, Taro S, Takashi Y, et al. Effects of coenzyme Q10 on salivary secretion. Clin Biochem. 2011;44:669-74.

7. Sekine K, Ota N, Nishii M, Uetake T, Shimadzu M. Estimation of plasma and saliva levels of coenzyme Q10 and influence of oral supplementation. Biofactors. 2005;25(1-4):205-11.

8. Dawood GA, Taqa GA, Alnema MM. Histological effects of Co Q10 on liver and buccal mucosa in mice. J Appl Vet Sci. 2020;5(2) 1-5.

9. Abdullah AG. Age related changes of submandibular salivary glands: An anatomical and histological study. Diyala J Med. 2011;1(1):53-61.

10. Deshmukh G, Venkataramaiah SB, Doreswamy CM, Umesh MC, Subbanna RB, Pradhan BK, et al. Safety assessment of ubiquinol acetate: Subchronic toxicity and genotoxicity studies. J Toxicol. 2019;2019:3680757.

11. Kitano M, Watanabe D, Oda S, Kubo H, Kishida H, Fujii K, et al. Subchronic oral toxicity of ubiquinol in rats and dogs. Int J Toxicol. 2008;27(2):189-215.

12. Inoue K, Morikawa T, Matsuo S, Tamura K, Takahashi M, Yoshida M. Adaptive parotid gland hypertrophy induced by dietary treatment of GSE in rats. Toxicol Pathol. 2014;42(6):1016-23.

13. Honda K, Tominaga S, Oshikata T, Kamiya K, Hamamura M, Kawasaki T, et al. Thirteen-week repeated dose oral toxicity study of coenzyme Q10 in rats. J Toxicol Sci. 2007;32(4):437-48. 
14. Daskala ID, Tesseromatis CC. Morphological changes of parotid gland in experimental hyperlipidemia. Int J Dent. 2011;2011:928386.

15. Veiga FF, Johann ACB, Kagy VS, Muniz LTB, Alanis LAR, Rosa EAR, et al. Action of lithium carbonate on parotid acini. Dent Oral Craniofac Res. 2016;2(3):287-91.

16. Mieliauskaite D, Venalis A, Graziene V, Kirdaite G. Bilateral parotid enlargement due to malnutrition under the influence of the media in an adolescent in Lithuania. Appetite. 2007;49(1):260-2.

17. Bertoldo BB, Bertoldo BB, Etchebehere RM, Furtado TCS, Faria JB, Silva CB, et al. Lingual salivary gland hypertrophy and decreased acinar density in chagasic patients without megaesophagus. Rev Inst Med Trop Sao Paulo. 2019;61:e67.
18. Yeroshenko GA, Fedoniuk LY, Shevchenko KV, Kramarenko DR, Yachmin AI, Vilkhova OV, et al. Structural reorganization of the rats' submandibular glands acini after the influence of $1 \%$ methacrylate. Wiad Lek. 2020;73(7):1318-22.

19. da Cunha Lima M, Sottovia-Filho D, Cestari TM, Taga R. Morphometric characterization of sexual differences in the rat sublingual gland. Braz Oral Res. 2004;18(1):53-8.

20. Raizner AE, Raizner AE. Coenzyme Q10. Methodist Debakey Cardiovasc J. 2019;15(3):185-91. 